\title{
A holding access-point assignment algorithm for IEEE802.11 wireless local-area networks
}

\section{Shigeto Tajima*}

Graduate School of Engineering Science, Osaka University,

Osaka, Japan

Email: tajima@ics.es.osaka-u.ac.jp

*Corresponding author

\section{Nobuo Funabiki}

Graduate School of Natural Science and Technology,

Okayama University,

Okayama, Japan

Email: funabiki@okayama-u.ac.jp

\section{Teruo Higashino}

Graduate School of Information Science and Technology,

Osaka University,

Osaka, Japan

Email: higashino@ist.osaka-u.ac.jp

\begin{abstract}
Nowadays, various types of access-points (APS) and hosts such as dedicated APs, laptop personal computers (PCs), and mobile terminals have been used in IEEE802.11 wireless local-area networks (WLANS). As a result, the optimal assignment of holding APs with different types into the network field, depending on the host type distribution, has become another important task to design high-performance WLANs. In this paper, we first define this holding access-point assignment problem as a combinatorial optimisation problem and propose its two-phase heuristic algorithm. Then, since plural partially overlapping channels are available in IEEE802.11 WLANs, we present the channel assignment extension to the APs such that the communication time of the APs is minimised, and the model to estimate the communication time increase by interferences. The effectiveness of our proposal is verified through simulations in six instances using the WIMNET simulator.
\end{abstract}

Keywords: wireless local-area network; WLAN; holding access-point; partially overlapping channel; assignment algorithm; combinatorial optimisation problem.

Reference to this paper should be made as follows: Tajima, S., Funabiki, N. and Higashino, T. (2018) 'A holding access-point assignment algorithm for IEEE802.11 wireless local-area networks', Int. J. Space-Based and Situated Computing, Vol. 8, No. 1, pp.50-58.

Biographical notes: Shigeto Tajima received his BS in Industrial Engineering from the Osaka Electro-Communication University, Japan, in 1992 and $\mathrm{PhD}$ in Information Science and Technology from the Osaka University, Japan, in 2010. From 1992 to 1995, he was in the System Engineering Division, NS and I System Service Corp., Japan. In 1995, he joined the Department of Information and Computer Sciences at the Osaka University, Japan, as an Experimental Officer, and became a Research Associate in 1996. His research interests include computer network and optimisation algorithm.

Nobuo Funabiki received his $\mathrm{BS}$ and $\mathrm{PhD}$ in Mathematical Engineering and Information Physics from the University of Tokyo, Japan, in 1984 and 1993, respectively. He received his MS in Electrical Engineering from the Case Western Reserve University, USA, in 1991. From 1984 to 1994, he was in Sumitomo Metal Industries, Ltd., Japan. He joined Department of Information and Computer Sciences at Osaka University, Japan, as an Assistant Professor in 1994, and moved to Department of Electrical and Communication Engineering at Okayama University as a Professor in 2001. His research interests include computer networks, optimisation algorithms, and web technology. He is a member of IEEE, IEICE, and IPSJ. 
Teruo Higashino received his $\mathrm{BS}, \mathrm{MS}$, and $\mathrm{PhD}$ in Information and Computer Science from the Osaka University, Japan in 1979, 1981 ad 1984, respectively. He joined the Faculty of Osaka University in 1984. Since 2002, he has been a Professor in Graduate School of Information Science and Technology at the Osaka University. His current research interests include design and analysis of distributed systems, communication protocol and mobile computing. He is a senior member of IEEE, a Fellow of IPSJ, and a member of ACM and IEICE.

This paper is a revised and expanded version of a paper entitled 'A Holding access-point assignment algorithm for wireless local-area networks' presented at IEEE 9th International Conference on Communication Software and Networks (ICCSN), Guangzhou, China, 6-8 May 2017.

\section{Introduction}

Recently, new access-point $(A P)$ devices providing higher performances and advanced functions have become available within short time for wireless local-area networks (WLANs), due to the rapid developments of the technologies for device manufacturing, wireless communications, and supporting software. As a result, many organisations including governments, companies, and schools have a variety of AP devices that have different communication performances and specifications, due to the implemented communication protocols and the adopted number of adopted multiple-input-multiple-output (MIMO) antennas. Currently, IEEE $802.11 \mathrm{~g}, 11 \mathrm{n}$, and 11 ac protocols have been practically used in WLANs. Among them, $11 \mathrm{n}$ and 11ac allows the use of MIMO antennas to increase the communication capacity by realising plural streams between the source and destination nodes using multiple antennas (Perahia and Stacey, 2008; Gast, 2013; Nee, 2011; Bejarano et al., 2013; Spencer et al., 2004; Hiraguri and Nishimori, 2015).

At the same time, the variation of client hosts for WLANs has been also increased. Traditionally, laptop personal computers (PCs) have been used as client hosts. In addition, smart phones and tablet terminals have often been used there. These hosts also support different protocols and have the different number of antennas.

Basically, the highest communication speed of a wireless link between an AP and a host can be confined by the lower specification of either device of the link. For example, when the AP supports IEEE802.11ac with three antennas and the host does IEEE 802.11n with two antennas, they can communicate with the lower specification of $11 \mathrm{n}$ with two antennas. In this case, the lower-spec AP supporting $11 \mathrm{n}$ with two antennas can be assigned there to provide the same communication speed, while the higher-spec AP supporting 11ac with three antennas should be assigned at other places where hosts supporting 11 ac are available. As a result, the proper assignment of the holding APs in the network field is another important task in designing high-performance WLANs, so that the wireless links between APs and hosts can perform with the highest specifications as best as possible.

In IEEE802.11 WLANs, a limited number of frequency channels are available. Actually, in Japan, only 13 channels can be used for $2.4 \mathrm{GHz}$ bands, and 19 channels can be for
$5 \mathrm{GHz}$ bands, when $20 \mathrm{MHz}$ band is allocated for one channel. The frequency bands of the adjacent channels are partially overlapping as shown in Figure 1 Cisco Systems, Inc. (2016), and thus, the links using close channels can cause interferences when they communicate at the same time. Thus, the proper channel assignment to the APs is also critical for high-performance WLANs.

Figure 1 Partially overlapping channels for $2.4 \mathrm{GHz}$ (see online version for colours)

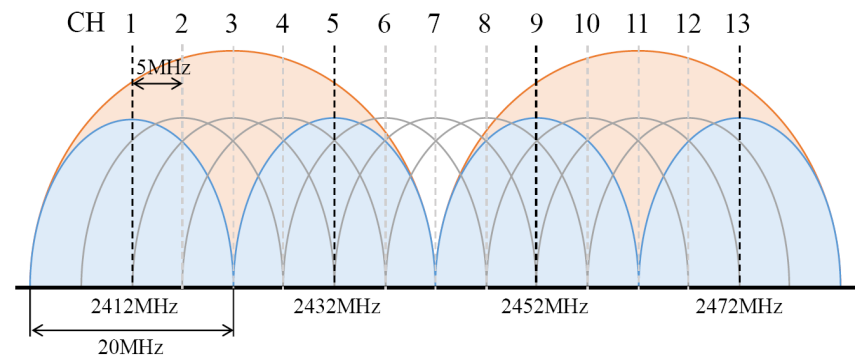

In this paper, first, we define the holding $A P$ assignment problem as a new combinatorial optimisation problem for designing high-performance WLANs, where the inputs, outputs, constraints, and objectives of the problem are formulated. In this formulation, it is assumed that the network layout information including the locations of the APs and the hosts is given as the inputs, and the link speed for any pair of an AP and a host can be calculated using the formula in Funabiki et al. (2012) from it. Besides, it is assumed that only one channel can be used for all the devices in the network for simplicity. Then, we propose the heuristic algorithm for this problem that consists of the greedy initialisation phase and the local search optimisation phase.

Next, to consider the optimal channel assignment to the APs, we present the channel assignment extension of the algorithm such that the communication time of the APs is minimised, and the model to estimate the communication time increase by the interference in partially overlapping channels. The effectiveness of our proposal is verified through simulations in simple instances using the WIMNET simulator.

The rest of this paper is organised as follows: Section 2 presents the holding AP assignment algorithm for WLANs Sections 3 and 4 present the channel assignment extension and the model for partially overlapping channels 
respectively. Section 5 shows the simulation results for evaluations. Section 6 provides concluding remarks with future works.

\section{Holding AP assignment algorithm}

In this section, we define the holding AP assignment problem and present its heuristic algorithm for high-performance WLANs.

\subsection{Device type}

The maximum throughput of a wireless communication link can be drastically changed, depending on the protocol, the frequency, and the number of antennas adopted in the devices of the end nodes of the link. In this paper, each combination of these factors is represented by a device type (or type) with a unique index number. Table 1 shows the eight types that are considered in this paper. The type with the larger index represents newer technologies that can provide higher performances than the type with the smaller index. Because of the lower type compatibility in WLAN devices, the maximum throughput of a link is determined by that of the device with the smaller index between the two end devices.

Table 1 Device type

\begin{tabular}{lcccc}
\hline Type & Protocol & Frequency & $\begin{array}{c}\text { \# of } \\
\text { antennas }\end{array}$ & $\begin{array}{c}\text { Max. speed } \\
(\text { Mbps })\end{array}$ \\
\hline 1 & $11 \mathrm{a}$ & 5.0 & 1 & 54 \\
2 & $11 \mathrm{~g}$ & 2.4 & 1 & 54 \\
3 & $11 \mathrm{n}$ & 2.4 & 1 & 150 \\
4 & $11 \mathrm{n}$ & 2.4 & 2 & 300 \\
5 & $11 \mathrm{n}$ & 2.4 & 3 & 450 \\
6 & $11 \mathrm{ac}$ & 5.0 & 1 & 433 \\
7 & $11 \mathrm{ac}$ & 5.0 & 2 & 867 \\
8 & $11 \mathrm{ac}$ & 5.0 & 3 & 1,300 \\
\hline
\end{tabular}

This paper assumes that the standard device type exists, such that the link speed change with the distance and the path obstacles between the end nodes is analysed using the measurement results of the devices with this type, and is well modelled. Using the link speed model for the standard type, the link speed for any type can be calculated. In this paper, the type 3 is actually selected as the standard one.

\subsection{Problem formulation}

In order to improve the performance of a WLAN, APs with larger indices should be assigned to the places in the network field where many hosts with larger indices exist, if the number of such APs is small among the holding ones. As a result, the optimal assignment of holding AP types becomes an important task to be solved. The optimal holding AP assignment problem can be defined as a combinatorial optimisation problem as follows:
- Input:

$H a_{k}$ the number of holding APs with type $k$

$H h_{k}$ the number of hosts with type $k$ in the field

$L a_{i}$ the $i^{\text {th }}$ AP location

$L h_{j} \quad$ the $j^{\text {th }}$ host in the field

$h t_{j} \quad$ the type of the $j^{\text {th }}$ host

$m_{k} \quad$ the maximum link speed of type $k$

$K \quad$ the type for the standard link speed.

- Output:

$a t_{i} \quad$ the assigned type to the $i^{\text {th }}$ AP location $L a_{i}$

$A h_{j} \quad$ the connecting AP placement of the $j^{\text {th }}$ host $L h_{j}$.

- Constraint:

1 The number of assigned APs with type $k$ must be equal to or less than $H a_{k}$.

2 Every host must be connected to one AP placement.

- Objective: to minimise the cost function $E$ in equation (1).

$$
E=A \sum_{i} \sum_{j \in A P_{i}} \frac{1}{s a_{i j}}+B \max _{i}\left[\sum_{j \in A P_{i}} \frac{1}{s a_{i j}}\right]
$$

where $A$ and $B$ represent the constant coefficients ( $A=5$ and $B=1$ in this paper), $A P_{i}$ does the set of hosts connected to $L a_{i}, \max _{i}[]$ returns the maximum value on $i, s a_{i j}$ does the link speed between AP $L a_{i}$ and host $L h_{j}$ that can be calculated by equation (2):

$$
s a_{i j}=s d_{i j} \frac{\min \left(m_{a t i}, m_{h t j}\right)}{m_{K}}
$$

where $s d_{i j}$ represents the standard link speed between $L a_{i}$ and $L h_{j}, m_{a t i}$ and $m_{h t j}$ do the maximum link speed of the AP assigned to $L a_{i}$ and the host to $L h_{j}$, and $m_{K}$ does the maximum link speed of the standard type. The $A$-term in $E$ represents the total delay time and the $B$-term does the maximum delay time of one AP when all the hosts are communicating at the same time.

\subsection{Algorithm}

The two-stage heuristic algorithm that is composed of the greedy method and the simulated annealing (SA) is presented for the holding AP assignment problem.

\subsubsection{Initial solution by greedy method}

In our algorithm, first, the initial solution is constructed by assigning the currently available AP with the highest performance or type index to the most congested unassigned AP location in the field.

1 Calculate the standard link speed $\left(s d_{i j}\right)$ for every pair of the AP location and the host, assuming the standard-type AP and host, by using the formula in 
Funabiki et al. (2012) from the layout information of the network.

2 Sort the holding APs in descending order of the maximum link speed.

3 Calculate the actual link speed $\left(s h_{i j}\right)$ for every pair of the AP location and the host considering their types by:

$s h_{i j}=s d_{i j} \frac{m_{h t j}}{m_{K}}$

4 Select the AP location that provides the largest maximum link speed in 3 for each host and associate the host to the corresponding AP location.

5 Count the number of hosts associated with each AP location in 4 and sorts the AP locations in descending order of this number.

6 Assign a holding AP to each AP location sequentially:

a Select the first unassigned AP location in the sorted list.

b Assign an unassigned AP whose type is equal to or larger than the type of any host associated with the selected AP location.

c If no such AP exists, assign an unassigned AP whose type has the minimum difference from the type of any host associated with the selected AP location.

7 Calculate the real link speed $s a_{i j}$ for each pair of an assigned AP and a host by equation (2).

8 Calculate the cost function $E$ in equation (1).

9 Save the solution as the best solution $S_{\text {best }}$ and the cost $E$ as the best cost $E_{\text {best }}$.

\subsubsection{Solution improvement by $S A$}

Next, the initial solution is iteratively improved by SA, where the following parameters are used:

$L_{\max }$ the local minimum convergence parameter ( $L_{\max }=10,000$ in the paper)

$R N$ the number of iterations $(R N=8,000,000)$

$T p \quad$ the SA temperature $(T p=0.00013)$.

1 Initialise the iteration counter $I_{c n t}$ and the local minimum counter $L_{c n t}$ by 0 .

2 Generate a neighbour solution from the current one.

a If $L_{c n t}<L_{\max }$, change the associated AP of a host by:

- Randomly select one host that can be associated with two or more APs.

- Randomly select one AP that is not currently associated but can be newly associated with the host. b Otherwise, swap the assigned APs between two AP locations by:

- Randomly select one AP location.

- Randomly select another AP location that is assigned an AP with the different type.

- Swap the assigned APs between the two AP locations.

3 Calculate the cost function $E^{\text {new }}$ for this neighbour solution and the increase of the cost function $\Delta E=E^{\text {new }}-E$ if the new solution is accepted.

4 If $\Delta \mathrm{E} \leq 0$ or rand $1 \leq \exp \left(-\frac{\Delta E}{T_{p}}\right)$, accept the neighbour solution as the new solution, where rand 1 returns a $0-1$ random number. Otherwise, discard it.

5 If $E^{\text {new }}<E_{\text {best }}$, update $S_{\text {best }}$ and $E_{\text {best }}$. Otherwise, increment $L_{c n t}$ by 1 .

6 If $I_{c n t}=R N$, output $S_{\text {best }}$ and terminate the algorithm. Otherwise, increment $I_{c n t}$ by 1 and go to 2 .

\section{Channel assignment extension}

In this section, we present the channel assignment extension for the holding AP assignment algorithm.

\subsection{Importance of channel assignment}

The IEEE802.11 standards allow the use of $2.4 \mathrm{GHz}$ and $5 \mathrm{GHz}$ bands with plural channels. Namely, the maximum of 13 channels in Japan are available for $2.4 \mathrm{GHz}$ and 19 channels for $5 \mathrm{GHz}$. The channels for $5 \mathrm{GHz}$ can be used at the same time without interferences. However, for the more popular $2.4 \mathrm{GHz}$, the allocated frequencies of the adjacent channels are partially overlapped so that the interferences can occur if they are used at the same time in the close locations. Actually, only four channels can be used without interference.

In addition, if the channel bonding is used for $11 \mathrm{n}$ or $11 \mathrm{ac}$, the number of non-overlapping channels further reduced because plural adjacent channels are used together as one channel (Deek et al., 2014; Wang et al., 2016; Hanada et al., 2013). Thus, the proper channel assignment to the APs is very important in designing high performance WLANs. In this section, we extend the holding AP assignment algorithm to consider the optimal channel assignment.

\subsection{Formulation of channel assignment extension}

The channel assignment extension of the holding AP assignment algorithm is formulated as follows:

- Inputs:

1 the holding AP assignment result: the type and connected hosts for each AP location 
$2|c|$ : the number of available non-overlapping channels

$3 R N_{c h}$ : the number of iterations $\left(R N_{c h}=1,000\right.$ in the paper)

$4 T p_{c h}$ : the SA temperature $\left(T p_{c h}=2.5\right)$

$5 \quad d_{w}$ : the interference range $\left(d_{w}=100\right)$.

- Outputs: $c_{i}$ : the assigned channel to $L a_{i}$.

- Objective: to minimise the following cost function $E_{c h}$ :

$$
\begin{aligned}
& E_{c h}=C \sum_{i} I T_{i}+D \max _{i}\left[I T_{i}\right] \\
& I T_{i}=\sum_{\substack{k \in I_{i} \\
c_{k}=c_{i}}} T_{k}
\end{aligned}
$$

where $I T_{i}$ represents the interference-aware communication time for $L a_{i}, T_{i}$ does the communication time for $L a_{i}, I_{i}$ does the set of the APs that can be interfered with $L a_{i}, C$ and $D$ do constant coefficients ( $C=1, D=4$ in this paper). This cost function intends the minimisation of the load of the overall network and the load of the bottleneck AP.

\subsection{Channel assignment procedure}

The channel assignment extension consists of three steps.

\subsubsection{AP interference graph}

In the first step, the AP interference graph $\left(G_{A P}\right)$ is generated to describe the radio interferences among the APs. Each vertex in $G_{A P}$ represents an AP and each edge does the interference between two end APs. In this paper, when two APs are located within the interference range $d_{w}$, the edge is generated, where the signal attenuations by obstacles between them is considered.

\subsubsection{Initial solution by greedy method}

The initial solution is constructed by assigning channels to the APs sequentially in descending order of the loads.

1 Calculate the communication time of each AP without considering the interferences from other APs by:

$$
T_{i}=\sum_{j} \frac{1}{s p_{i j}}
$$

where $T_{i}$ represents the communication time for $L a_{i}$ and $s p_{i j}$ does the link speed between $L a_{i}$ and host $j$.

2 Calculate the adjacent communication time for each AP by:

$$
N T_{i}=\sum_{e(i, k) \in G_{A P}} T_{k}
$$

where $N T_{i}$ represents the adjacent communication time for $L a_{i}$ and $e(i, k)$ represents the edge between $L a_{i}$ and $L a_{k}$.
3 Sort the APs in descending order of $N T_{i}$ where the tiebreak is resolved by $T_{i}$.

4 Generate the set of the interfered APs for each AP by:

a Initialise the interfered AP set $I_{i}$ for $L a_{i}$ by $L a_{i}$ itself: $I_{i}=\{i\}$.

b Add the AP that is interfered with every AP in the current $I_{i}$ into $I_{i}$ in the sorted order.

5 Calculate the interference communication time for $L a_{i}$ by:

$$
A T_{i}=\sum_{k \in I_{i}} T_{k}
$$

where $A T_{i}$ represents the interference communication time for $L a_{i}$.

6 Sort the APs in descending order of $A T_{i}$ where the tiebreak is resolved by $N T_{i}$.

7 Assign one channel to each AP in the sorted order such that the following channel communication time is minimised:

$$
I T_{i c}=\sum_{\substack{k \in I_{i} \\ c_{k}=c}} T_{k}
$$

where $I T_{i c}$ represents the channel communication time for $L a_{i}$ with channel $c$. It is noted that one channel for each of $2.4 \mathrm{GHz}$ and $5.0 \mathrm{GHz}$ must be assigned here if the AP can use both bands.

8 Save the initial solution as the best solution $S_{\text {best }}$ and the initial cost $E_{c h}$ as the best cost $E_{\text {best }}$.

\subsubsection{Solution improvement by $S A$}

As in the holding AP assignment, the initial solution is improved by SA.

1 Initialise the iteration counter $I_{c n t}$ by 0 .

2 Randomly select one AP to change its assigned channel.

3 Randomly select a new channel to the AP.

4 Calculate the new cost function $E_{c h}^{\text {new }}$ for this channel assignment and the cost function increase by $\Delta E_{c h}=E_{c h}^{n e w}-E_{c h}$.

5 If $\Delta E_{c h} \leq 0$ or rand $1 \leq \exp \left(-\frac{\Delta E_{c h}}{T p_{c h}}\right)$, accept the new channel. Otherwise, discard it.

6 If $E_{c h}^{\text {new }}<E_{\text {best }}$, update the best solution $S_{\text {best }}$.

7 If $I_{c n t}=R N_{c h}$, output $S_{\text {best }}$ and terminate the algorithm. Otherwise, increment $I_{c n t}$ by 1 and go to 2 . 


\section{Model for partially overlapping channel}

In this section, we present a simple model to estimate the link speed drop or the communication time increase caused by the interferences in partially overlapping channels.

\subsection{Interference degree}

For this model, the interference degree between two adjacent APs is defined to estimate the communication time increase of each incident link to them. Actually, it is represented by the channel interference degree that is given by the difference between the channels assigned to the APs, and distance interference degree that is given by the physical distance between the AP locations.

\subsubsection{Channel interference degree}

Zhou et al. (2014) presented the channel interference degree between two IEEE802.11n links using the $20 \mathrm{MHz}$ channel width without the channel bonding. Because our paper considers the $40 \mathrm{MHz}$ channel width by the channel bonding, we extended their channel interference degrees by taking the average of the degrees for two adjacent $20 \mathrm{MHz}$ channels. For example, when the channel difference is 1 , one $20 \mathrm{MHz}$ channel among the bonded $40 \mathrm{MHz}$ channel is partially overlapped by the adjacent $20 \mathrm{MHz}$ channel, and another one is fully overlapped. Thus, in this case, the channel interference degree becomes $(0.7272+1.0) / 2=$ 0.8636 , where 0.7272 is given as the degree between the two adjacent $20 \mathrm{MHz}$ channels in Zhou et al. (2014). Table 2 shows the channel interference degree for each channel difference. When the channel difference is 0 , it becomes the largest 1 , and when it is larger than 8 , it becomes 0 . Then, our model assumes that the communication time of a link is proportionally increased by this degree.

\subsubsection{Distance interference degree}

The distance interference degree is decreased proportionally to the physical distance $d$ between the locations of the APs, as shown in the following equation:

$$
f(d)=\max \left(0,1-d / d_{w}\right)
$$

where $d_{w}$ represents the maximum range of the interference, where $d_{w}=100$ is used in this paper.

\subsection{Communication time amendment by interference degree}

The communication time $T_{i}$ of $A P_{i}$ should be amended to reflect the interferences from the APs within its interference range. The interference degree $i d_{i k}$ of $A P_{i}$ from $A P_{k}$ is calculated by the multiplication of the channel interference degree $c d_{i k}$ and the distance interference degree $d d_{i k}$. It is noted that $c d_{i k}$ can be obtained from Table 2 by considering the difference between the assigned channels, and $d d_{i k}$ can be from equation (10) using the physical distance between their locations. Our model assumes that the communication time is proportionally increased by the summation of the interference degrees from its interfered APs:

$$
\begin{aligned}
& i d_{k}=c d_{i k} \times d d_{i k} \\
& T_{i}^{i n t}=T_{i} \times\left(1+\sum_{k \neq 1} i d_{i k}\right)
\end{aligned}
$$

where $T_{i}^{\text {int }}$ represents the amended communication time of $L a_{i}$ by the interferences from other APs.

In the channel assignment extension in Section 3, this amended communication time is used to calculate the new cost function $E_{c h}^{\text {new }}$ in the solution improvement by SA in Section 3.3.3, where all the APs are assigned channels, and the interference degrees among them can be calculated.

\subsection{Discussion of interference model under partially overlapping channels}

Here, we discuss related studies on the interference model under partially overlapping channels. Alim Al Islam et al. (2016) investigated the channel assignment method in wireless mesh networks where each node has multiple wireless communication interfaces. Wang and Shi (2016), proposed the assignment method of end-to-end channels assignment using partially overlapping channels for wireless mesh networks. They referred the work in Mishra et al. (2006) for partially overlapping channels. Duarte et al. (2012) proposed the channel assignment algorithm for wireless mesh networks using the game theory. Ding et al. (2012) proposed the genetic algorithm to improve the throughput using partially overlapping channels. Jaumard et al. (2016) studied throughput improvements and delay reductions in wireless mesh networks using partially overlapping channels. Zhao et al. (2014) proposed the algorithm for allocating APs and assigning partially overlapping channels using the mixed integer liner programming (MILP) for wireless networks. Zhao et al. (2016) also proposed the algorithm for allocating AP densities and assigning partially overlapping channels. Wang et al. (2015) proposed the channel assign algorithm using partially overlapping channels for multi-channel multi-radio wireless mesh networks.

Among them, the studies in Wang and Shi (2016), Mishra et al. (2006), Jaumard et al. (2016), and Wang et al. (2015) determine the interference degree by calculating the signal strength. The studies in Duarte et al. (2012) and Ding et al. (2012) use measurement results. Their results show that interference degrees are different from each other. 
Table 2 Channel interference degree $(40 \mathrm{MHz})$

\begin{tabular}{llcccccccc}
\hline Channel difference & 0 & 1 & 2 & 3 & 4 & 5 & 6 & 7 & 8 \\
Interference degree & 1 & 0.8636 & 0.6357 & 0.51875 & 0.5027 & 0.364 & 0.1358 & 0.01875 & 0.0027 \\
\hline
\end{tabular}

Table 3 Types and numbers of holding APs and hosts in three instances

\begin{tabular}{lcc}
\hline Instance & AP: type (number) & Host: type (number) \\
\hline 1 & $7(5), 4(5)$ & $50: 7(25), 4(25)$ \\
& $75: 7(38), 4(37)$ \\
& $100: 7(50), 4(50)$ \\
2 & $4(5), 3(5)$ & $50: 4(25), 3(25)$ \\
& $75: 4(38), 3(37)$ \\
& $100: 4(50), 3(50)$ \\
3 & $8(2), 7(2), 6(1)$, & $50: 7(25), 4(25)$ \\
& $5(2), 4(2), 3(1)$ & $75: 7(38), 4(37)$ \\
& & $100: 7(50), 4(50)$ \\
\hline
\end{tabular}

\section{Evaluations by simulations}

In this section, we evaluate our proposal through simulations using the WIMNET simulator Funabiki (2011), in both cases with/without partially overlapping channels.

\subsection{Case without partially overlapping channels}

First, we evaluate the proposed algorithm for simple cases without using partially overlapping channels.

\subsubsection{Simulation instances}

In the simulation instances, the square plain field of $150 \mathrm{~m} \times 50 \mathrm{~m}$ is used, where 10 AP locations are selected along the boundary with the same interval, and 50,75 , or 100 hosts are distributed randomly. Table 3 summarises the types of APs and the hosts with their numbers. As the number of non-overlapping channels, four is used for $5.0 \mathrm{GHz}$ and two is for $2.4 \mathrm{GHz}$. The standard link speed $s d_{i j}$ for any pair of an AP and a host is calculated from their coordinates and the link speed estimation equation in Funabiki et al. (2012).

\subsubsection{Algorithm result for instance 1}

In Figure 2, the type-4 AP is connected with only type-4 hosts and the type-7 AP is with type- 4 or type-7 hosts. The number of associated hosts for the type- 4 AP is three or less, and that for the type-7 AP is between six and nine. As the result, the communication time for the AP exists between 0.006 and 0.029 . This result indicates that the AP assignment and host association result by the algorithm does not only satisfy the constraints but also avoids the bottleneck AP.
Figure 2 AP and channel assignment result for instance 1

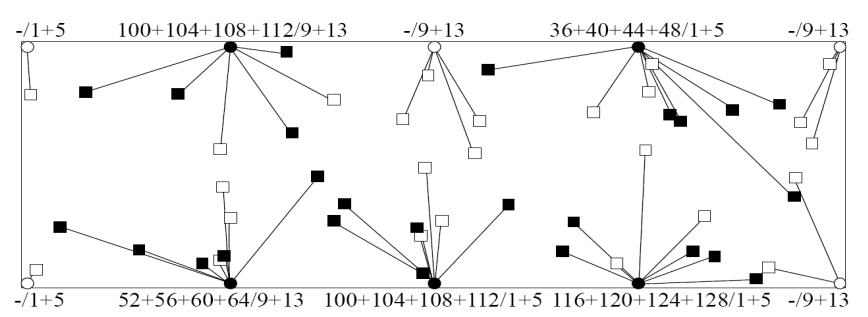

\subsubsection{Throughput results}

Table 4 shows the throughput results for the three instances using the WIMNET simulator. Here, the four cases for the holding AP and channel assignments are considered:

$1 \mathrm{AP} /$ channel assignment by proposed algorithm using four channels for $5.0 \mathrm{GHz}$ and two channels for $2.4 \mathrm{GHz}$ (proposal).

2 AP assignment by congestion order using one channel (compare 1).

3 AP assignment by proposed algorithm using one channel (compare 2).

4 AP assignment by proposed algorithm and random channel assignment using four channels for $5.0 \mathrm{GHz}$ and two channels for $2.4 \mathrm{GHz}$ (compare 3 ).

In 2, the APs are sorted in descending order of the number of the nearest hosts, and the holding APs are assigned there in this order from the highest performance ones.

Table 4 shows that the throughput for compare 2 is about 1.1 times higher than that for compare 1. It indicates that the AP assignment in the proposed algorithm can improve the holding AP assignment. Besides, they show that the throughput for proposal is about 1.3 times higher than that for compare 3 .

\subsubsection{Convergence of SA for channel assignment extension}

In this subsection, we investigated the convergence of SA for the channel assignment extension to better network solutions. Figure 3 shows the value of the cost function $E_{c h}$ and the simulated throughput (Mbps) of the final solution by SA for instance 1 with 50 hosts, when the number of iterations $R N_{c h}$ is set $0,100,500$, and 1,000. It indicates that $E_{c h}$ decreases and the throughput increases until $R N_{c h}=500$. Thus, the convergence of SA to better solutions with smaller values of $E_{c h}$ indicates the convergence of the network to better performances with higher throughputs. 
Table 4 Throughput results

\begin{tabular}{lccccc}
\hline \multirow{2}{*}{ Instance } & \# of & \multicolumn{4}{c}{ Throughput (Mbps) } \\
\cline { 3 - 6 } & hosts & Proposal & Compare1 & Compare2 & Compare3 \\
\hline 1 & 50 & 658.10 & 272.90 & 295.80 & 521.57 \\
& 75 & 615.07 & 244.10 & 294.78 & 471.83 \\
& 100 & 569.25 & 243.11 & 268.25 & 414.92 \\
2 & 50 & 181.10 & 95.49 & 97.24 & 120.32 \\
& 75 & 200.82 & 96.67 & 97.68 & 170.39 \\
& 100 & 181.21 & 92.03 & 96.80 & 145.35 \\
3 & 50 & 656.06 & 245.54 & 298.85 & 482.60 \\
& 75 & 618.42 & 223.19 & 293.29 & 480.58 \\
& 100 & 544.39 & 212.72 & 265.87 & 466.26 \\
\hline
\end{tabular}

Figure 3 Relationship $E_{c h}$ and throughput for instance 1, 50 hosts (see online version for colours)

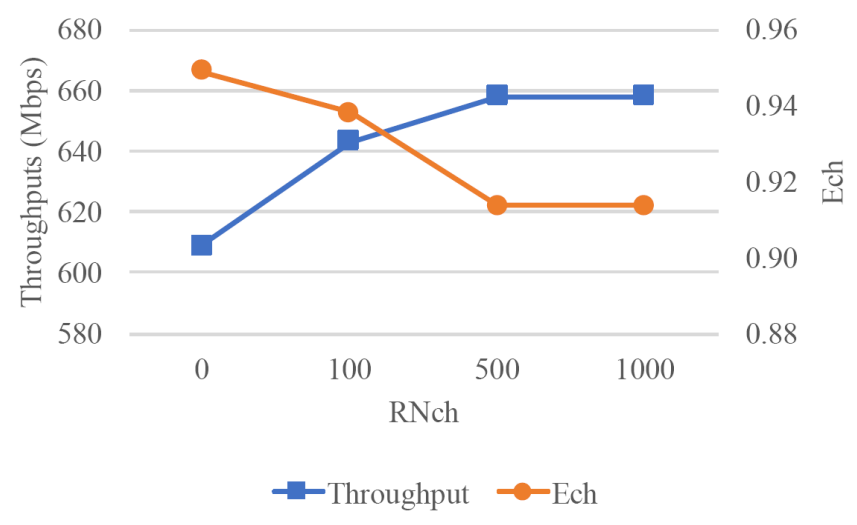

\subsection{Case with partially overlapping channels}

Next, we evaluate the proposed algorithm for more realistic cases using partially overlapping channels. For simulations by the WIMNET simulator, the following amended link speed is used for the link speed:

$$
s p_{i j}^{i n t}=s p_{i j} /\left(1+\sum_{\substack{k \\ k \neq 1}} i d_{i k}\right)
$$

where $s p_{i j}^{i n t}$ represents the amended link speed of each link incident to $A P_{i}$ by the interferences from other APs.

\subsubsection{Simulation instances}

In the simulated instances, the square plain field of $150 \mathrm{~m} \times 50 \mathrm{~m}$ for instance 4 , the field of $75 \mathrm{~m} \times 25 \mathrm{~m}$ for instance 5 as the half size of instance 4 , and the field of $300 \mathrm{~m} \times 100 \mathrm{~m}$ for instance 6 as the double size, are used. In each field, 10 AP locations are selected with the same interval along the top and bottom boundaries, and 50 hosts are randomly allocated. For simplicity, all the APs have type 4 (11n and two antennas), and all the hosts have type 3 ( $11 \mathrm{n}$ and one antenna). To avoid the bias in random numbers, five trials are repeated for each instance using different seeds, and their average results are used in evaluations.

\subsubsection{Algorithm results}

Figure 4 illustrates the channel assignment result using partially overlapping channels for instance 4 . The channel bonding is adopted here. The black circle represents an AP and the white square does a host. This figure shows that each AP is associated with the similar number of hosts in order to average the loads among them. Besides, among 13 channels, eight bonding channels of $1+5,2+6,3+7$, $4+8,6+10,7+11,8+12$, and $9+13$ are used here.

\subsubsection{Throughput results}

Table 5 shows the total throughput results by the proposed algorithm using three partially overlapping channels for the three instances. For the comparison, the results using two non-overlapping channels of $1+5$ and $9+13$ are also shown there.

Table 5 Throughput results using eight partially overlapping channels

\begin{tabular}{lcc}
\hline \multirow{2}{*}{ Instance } & \multicolumn{2}{c}{ Throughput (Mbps) } \\
\cline { 2 - 3 } & Proposal & Compare \\
\hline 4 & 156.63 & 66.79 \\
5 & 98.46 & 41.81 \\
6 & 270.33 & 203.92 \\
\hline
\end{tabular}

Table 5 indicates that the proposal improves the throughput by 2.4 times for instance 4 , by 2.4 times for instance 5 , and by 1.3 times for instance 6 from the comparison, which confirms the effectiveness of using partially overlapping channels in the proposal. Particularly, the improvement is largest for instance 5 that has the smallest field. In this instance, since every AP is located within the interference range, only two APs assigned different channels can communicate at the same time in the comparison. On the other hand, three APs can communicate at the same time in the proposal, although the link speed is decreased by the interference. These results suggest that the use of partially overlapping channels is more effective in congested fields with a lot of APs.

\subsection{Effect of interference model accuracy}

In this subsection, we discuss the effect of the accuracy of the interference model under partially overlapping channels to the throughput performance of the network. This model considers the channel interference degree and the distance interference degree to estimate the link speed drop by interferences from other links under partially overlapping channels. In this subsection, we prepare a reference case where both the degrees are increased by $R$ times and are saturated by 1.0. The three cases of $R=1.1,1.2,1.3,1.4$, and 1.5 are examined. Table 6 shows the throughput results for these three reference cases. 
Table 6 Throughput results for reference cases.

\begin{tabular}{lccccc}
\hline \multirow{2}{*}{ Instance } & \multicolumn{5}{c}{ Throughput (Mbps) } \\
\cline { 2 - 6 } & $R=1.1$ & $R=1.2$ & $R=1.3$ & $R=1.4$ & $R=1.5$ \\
\hline 4 & 139.15 & 125.39 & 115.57 & 106.73 & 99.64 \\
5 & 84.58 & 74.31 & 67.61 & 62.62 & 58.76 \\
6 & 255.04 & 240.85 & 228.40 & 216.35 & 204.96 \\
\hline
\end{tabular}

The results in Table 6 are better than the throughput results in 'compare' in Table 5 in any instance. It indicates that our proposal with the interference model under partially overlapping channels can improve the throughput performance of the network, even if the model accuracy is not sufficient. The accuracy investigation will be in future works.

\section{Conclusions}

This paper defines the holding access-point (AP) assignment problem as a new optimisation one for designing high-performance WLANs and presents its two-phase heuristic algorithm. The channel assignment extension to the APs using partially overlapping channels is also presented with the model for estimating the link speed drop by interferences. The effectiveness of our proposal is verified through simulations in six instances with and without partially overlapping channels. In future works, the performance of our proposal will be evaluated in a variety of simulation instances that have non-uniform host distributions and consider indoor environments.

\section{References}

Alim Al Islam, A.B.M., Islam, M.J., Nurain, N. and Raghunathan, V. (2016) 'Channel assignment techniques for multi-radio wireless mesh networks: a survey', IEEE Commun. Surveys \& Tutorials, Vol. 18, No. 2, pp.988-1017.

Bejarano, O., Knightly, E.W. and Park, M. (2013) 'IEEE 802.11ac: from channelization to multi-user MIMO', IEEE Commun. Magazine, October, Vol. 51, No. 10, pp.84-90.

Cisco Systems, Inc. (2016) Enterprise Mobility 8.1 Design Guide, November [online] http://www.cisco.com/c/en/us/td/docs/ wireless/controller/8-1/Enterprise-Mobility-8-1-DesignGuide/Enterprise_Mobility_8-1_Deployment_Guide.html (accessed 1 April 2017).

Deek, L., Garcia-Villegas, E., Belding, E., Lee, S.J. and Almeroth, K. (2014) 'Intelligent channel bonding in 802.11n WLANs', IEEE Trans. on Mobile Compu., June, Vol. 13, No. 6, pp.1242-1255.

Ding, Y., Huang, Y., Zeng, G. and Xiao, L. (2012) 'Using partially overlapping channels to improve throughput in wireless mesh networks', IEEE Trans. Mobile Comput., November, Vol. 11, No. 11, pp.1720-1733.

Duarte, P.B.F., Fadlullah, Z.M., Vasilakos, A.V. and Kato, N. (2012) 'On the partially overlapped channel assignment on wireless mesh network backbone: a game theoretic approach', IEEE J. Sel. Area. Commu., January, Vol. 30, No. 1, pp.119-127.
Funabiki, N. (Ed.) (2011) Wireless Mesh Networks, InTech, Open Access Publisher [online] https://www.intechopen.com/ books/editor/wireless-mesh-networks.

Funabiki, N., Maruyama, W., Nakanishi, T. and Watanabe, K. (2012) 'An extension of routing tree algorithm considering link speed change in IEEE $802.11 \mathrm{n}$ protocol for wireless mesh network', Proc. MENS2012, September, pp.600-605.

Gast, M. (2013) 802.11ac: A Survival Guide, O'Reilly Media, July [online] http://chimera.labs.oreilly.com/books/ 1234000001739/index.html (accessed 1 April 2017).

Hanada, K., Yamamoto, K., Morikura, M., Ishihara, K. and Kudo, R. (2013) 'Game-theoretic analysis of multibandwidth channel selection by coordinated APs in WLANs', IEICE Trans. on Commun., June, Vol. E96-B, No. 6, pp.1277-1287.

Hiraguri, T. and Nishimori, K. (2015) 'Survey of transmission methods and efficiency using MIMO technologies for wireless LAN Systems', IEICE Trans. Commun., July, Vol. E98-B, No. 7, pp.1250-1267.

Jaumard, B., Negahishirazi, M. and Kaddour, M. (2016) 'Increased throughput and reduced delay with partially overlapping channel in WMNs', Proc. Elect. Comp. Eng., May, pp.1-6.

Mishra, A., Shrivastava, V., Banerjee, S. and Arbaugh, W. (2006) 'Partially overlapped channels not considered harmful,' $A C M$ SIGMETRICS Perform. Eva. Review, Vol. 34, No. 1, pp.6374.

Nee, R.V. (2011) 'Breaking the gigabit-per-second barrier with 802.11ac', IEEE Wireless Commun., April, Vol. 18, No. 2, p.4.

Perahia, E. and Stacey, R. (2008) Next Generation Wireless LANs: Throughput, Robustness, and Reliability in 802.11n, Cambridge Univiversity Press, Cambridge, August.

Spencer, Q.H., Peel, C.B., Swindlehurst, A.L. and Haardt, M. (2004) 'An introduction to the multi-user MIMO downlink', IEEE Commun. Magazine, October, Vol. 42, No. 10, pp.60-67.

Wang, J. and Shi, W. (2016) 'Partially overlapped channels- and flow-based end-to-end channel assignment for multi-radio multi-channel wireless mesh networks', China Commun., April, Vol. 13, No. 4, pp.1-13.

Wang, J.H., Shi, W.X., Cui, K.Q., Jin, Fe. and Li, Y.X. (2015) 'Partially overlapped channel assignment for multi-channel multi-radio wireless mesh networks', Eurasip J. on Wireless Commun. and Netw., December, No. 1, p.25.

Wang, W., Zhang, F. and Zhang, Q. (2016) 'Managing channel bonding with clear channel assessment in 802.11 networks', Proc. IEEE Int. Conf. on Commun. (ICC), May, p.1-6.

Zhao, W., Fadlullah, Z., Nishiyama, H., Kato, N. and Hamaguchi, K. (2014) 'On joint optimal placement of access points and partially overlapping channel assignment for wireless networks', Proc. Global Commun. Conf., December, pp.4922-4927.

Zhao, W., Nishiyama, H., Fadlullah, Z., Kato, N. and Hamaguchi, K. (2016) 'DAPA: capacity optimization in wireless networks through a combined design of density of access points and partially overlapped channel allocation', IEEE Trans. Veh. Technol., May, Vol. 65, No. 5, pp.3715-3722.

Zhou, K., Jia, X., Chang, Y. and Tang, X. (2014) 'Partially overlapping channel assignment for WLANs using SINR interference model', Int. J. Commun. Syst., November, Vol. 27, No. 11, pp.3082-3095. 